\title{
New Approach of Stability for Time-Delay Takagi-Sugeno Fuzzy System Based on Fuzzy Weighting-Dependent Lyapunov Functionals
}

\author{
Yassine Manai, Mohamed Benrejeb, Pierre Borne \\ L.A.R.A. Automatique, Ecole Nationale d'Ingénieurs de Tunis, Le Belvédère, Tunis, Tunisie \\ E-mail:yacine.manai@gmail.com,mohamed.benrejeb@enit.rnu.tn,p.borne@ec-lille.fr \\ Received February 19, 2011; revised May 25, 2011; accepted June 3, 2011
}

\begin{abstract}
This paper deals with the stability of Takagi-Sugeno fuzzy models with time delay. Using fuzzy weightingdependent Lyapunov-Krasovskii functionals, new sufficient stability criteria are established in terms of Linear Matrix Inequality; hence the stability bound of upper bound delay time can be easily estimated. Finally, numeric simulations are given to validate the developed approach.
\end{abstract}

Keywords: Takagi-Sugeno Fuzzy Models, Linear Matrix Inequalities LMIs, Fuzzy Weighting-Dependent Lyapunov-Krasovskii Functionals

\section{Introduction}

Fuzzy control systems have experienced a big growth of industrial applications in the recent decades, because of their reliability and effectiveness. Many researches are investigated on the Takagi-Sugeno fuzzy models [1-17] which can associate the flexible fuzzy logic theory and rigorous mathematical theory into a unified framework. Thus, it becomes a powerful tool in approximating a complex nonlinear system.

Recently, Takagi-Sugeno fuzzy model approach has been used to examine nonlinear systems with time-delay, and different methodologies have been proposed for analysis and synthesis of this type of systems [1,2]. Time delay often occurs in many dynamical systems such as biological systems, chemical system, metallurgical processing system and network system. Their existences are frequently a cause of infeasibility and poor performances.

The developed stability approaches can be classified into two types. The first one is called as the delay independent stability criteria which do not include any information about the size of the time delay, whereas the second is called as the delay dependent stability criteria, in which the size of the time delay is taken explicitly in the formulation. It is generally recognized that delay dependent results are usually less conservative than delay independent ones, especially when the size of delay is small.

Two classes of Lyapunov-Krasovskii functionals are used to analysis these systems: quadratic LyapunovKrasovskii functionals and non-quadratic LyapunovKrasovskii ones. The use of first class brings much conservativeness in the stability test. In order to reduce the conservatism entailed in the previous results using quadratic functionals, a fuzzy weighting dependent approach is presented recently in [3] for fuzzy systems with fuzzy weighting functions.

In this paper, a new stability conditions for time-delay Takagi-Sugeno fuzzy systems by using fuzzy weightingdependent Lyapunov-Krasovskii functionals are presented. We derive delay-dependent stability conditions using recent technique called free-weighting matrix method [4]. This criterion is expressed in terms of Linear Matrix Inequalities (LMIs) which can be efficiently solved by using various convex optimization algorithms $[6,15]$. The proposed approach improves existing ones in [4].

The organization of the paper is as follows. In Section 2 , we present the system description and problem formulation and we give some preliminaries which are needed to derive results. Section 3 will be concerned to stability analysis for time-delay T-S fuzzy systems. Illustrative example is given in Section 4 for a comparison of previous results to demonstrate the advantage of proposed method. Finally Section 5 makes conclusion. 
Notation: Throughout this paper, a real symmetric matrix $S>0$ denotes $S$ being a positive definite matrix. The superscript " $T$ " is used for the transpose of a matrix. And where an ellipsis "*” denotes a block induced easily by symmetry.

\section{System Description and Preliminaries}

Consider a T-S fuzzy continuous model with time-delay for a nonlinear system as follows:

$$
\begin{aligned}
& \text { If } z_{1}(t) \text { is } M_{i 1} \text { and } \cdots \text { and } z_{p}(t) \text { is } M_{i p} \\
& \text { Then }\left\{\begin{array}{l}
\dot{x}(t)=A_{i} x(t)+A_{\tau i} x(t-\tau(t)) \\
x(t)=\phi(t), t \in\left[\tau_{0}, 0\right]
\end{array}\right.
\end{aligned}
$$

where $M_{i j}(i=1,2, \cdots, r, j=1,2, \cdots, p)$ is the fuzzy set and $\mathrm{r}$ is the number of model rules; $x(t) \in \mathfrak{R}^{n}$ is the state vector, $u(t) \in \mathfrak{R}^{m}$ is the input vector, $A_{i} \in \mathfrak{R}^{n \times n}$, $A_{\tau i} \in \mathfrak{R}^{n \times n}$ are constant real matrices, and $z_{1}(t), \cdots, z_{p}(t)$ are known premise variables, $\phi(t)$ is a continuous vector-valued initial function on $\left[-\tau_{0}, 0\right]$; the time-delay $\tau(t)$ may be unknown but is assumed to be smooth function of time.

$$
0 \leq \tau(t) \leq \tau_{0}, \quad \dot{\tau}(t) \leq d \prec 1,
$$

where $\tau_{0} \succ 0$ and $d$ are two scalars.

The final outputs of the fuzzy systems are:

$$
\begin{gathered}
\dot{x}(t)=\sum_{i=1}^{r} h_{i}(z(t))\left\{A_{i} x(t)+A_{\tau i} x(t-\tau(t))\right\} \\
x(t)=\phi(t), \quad t \in\left[-\tau_{0}, 0\right],
\end{gathered}
$$

where

$$
\begin{gathered}
z(t)=\left[z_{1}(t) z_{2}(t), \cdots, z_{p}(t)\right] \\
h_{i}(z(t))=w_{i}(z(t)) / \sum_{i=1}^{r} w_{i}(z(t)), \\
w_{i}(z(t))=\prod_{j=1}^{p} M_{i j}\left(z_{j}(t)\right) \text { for all } t .
\end{gathered}
$$

The term $M_{i 1}\left(z_{j}(t)\right)$ is the grade of membership of $z_{j}(t)$ in $M_{i 1}$

$$
\text { Since }\left\{\begin{array}{l}
\sum_{i=1}^{r} w_{i}(z(t)) \succ 0 \\
w_{i}(z(t)) \geq 0, \quad i=1,2, \cdots, r
\end{array}\right.
$$

we have

$$
\left\{\begin{array}{l}
\sum_{i=1}^{r} h_{i}(z(t))=1 \\
h_{i}(z(t)) \geq 0, \quad i=1,2, \cdots, r
\end{array} \quad \text { for all } t .\right.
$$

The time derivative of premise membership functions is given by:

$$
\dot{h}_{i}(z(t))=\frac{\partial h_{i}}{\partial z(t)} \times \frac{\partial z(t)}{\partial x(t)} \times \frac{\mathrm{d} x(t)}{\mathrm{d} t}=\sum_{l=1}^{s} v_{i l} \xi_{i l} \times \frac{\mathrm{d} x(t)}{\mathrm{d} t}
$$

We have the following property:

$$
\sum_{k=1}^{r} \dot{h}_{k}(z(t))=0 .
$$

\section{Assumption 1}

The time derivative of the premises membership function is upper bounded such that $\left|\dot{h}_{k}\right| \leq \beta_{k}$, for $k=1, \cdots, r$, where, $\beta_{k}, k=1, \cdots, r$ are given positive constants.

\section{Lemma 1[4]}

Under assumption 1, the time-delay Takagi-Sugeno fuzzy system is stable if there exist positive definite symmetric matrices $P_{j} \succ 0, \quad Q \succ 0, \quad Z \succ 0, \quad Y_{j}$ and $T_{j}, \quad j=1,2, \cdots, r$, such that the following LMIs hold.

$$
\begin{aligned}
& P_{k} \geq P_{r}, \quad k \in\{1, \cdots, r-1\} \\
& \Omega_{i j}+\Omega_{j i} \prec 0, \quad i \leq j,
\end{aligned}
$$

where

$$
\Omega_{i j}=
$$

$$
\left[\begin{array}{cccc}
\sum_{k=1}^{r-1} \beta_{k}\left(P_{k}-P_{r}\right) & & & \\
+P_{j} A_{i}+A_{i}^{T} P_{j} & P_{j} A_{\tau i}-Y_{j}+T_{j}^{T} & \tau_{0} A_{i}^{T} Z & -\tau_{0} Y_{j} \\
+Y_{j}+Y_{j}^{T}+Q & & & \\
* & -T_{j}-T_{j}^{T}-(1-d) Q & \tau_{0} A_{\tau i}^{T} Z & -\tau_{0} T_{j} \\
* & * & -\tau_{0} Z & 0 \\
* & * & * & -\tau_{0} Z
\end{array}\right]
$$

\section{Lemma 2[4]}

Under assumption1, system (2) with $d=0$ is asymptotically stable if there exist matrices $P_{j} \succ 0, W_{j} \succ 0$, $S_{j} \geq 0, \quad Q \succ 0, \quad U, \quad R \succ 0, \quad Z \succ 0, \quad Y_{j}, \quad T_{j}$ and $N_{j}, \quad j=1,2, \cdots, r$, such that the following LMIs hold:

$$
\begin{gathered}
{\left[\begin{array}{cc}
P_{k} & W_{k} \\
* & S_{k}
\end{array}\right] \geq\left[\begin{array}{cc}
P_{r} & W_{r} \\
* & S_{r}
\end{array}\right], k=1,2, \cdots, r-1,} \\
{\left[\begin{array}{cc}
Q & U \\
* & R
\end{array}\right] \geq 0,} \\
\Psi_{i j}+\Psi_{j i} \prec 0, \quad i \leq j,
\end{gathered}
$$

where

$$
\Psi_{i j}=
$$

$$
\left[\begin{array}{ccccc}
\Psi_{i j}^{(11)} & \Psi_{i j}^{(12)} & A_{i}^{T}\left(R+\tau_{0} Z\right) & W_{j}+N_{j}^{T} & -\tau_{0} Y_{j} \\
* & \Psi_{i j}^{(22)} & A_{\tau_{i}}^{T}\left(R+\tau_{0} Z\right) & S_{j}-N_{j}^{T}-U & -\tau_{0} T_{j} \\
* & * & -R-\tau_{0} Z & 0 & 0 \\
* & * & * & -R & -\tau_{0} N_{j} \\
* & * & * & * & -\tau_{0} Z
\end{array}\right],
$$




$$
\begin{gathered}
\Psi_{i j}^{(11)}=\sum_{k=1}^{r-1} \beta_{k}\left(P_{k}-P_{r}\right)+\left(P_{j}+U\right) A_{i} \\
+A_{i}^{T}\left(P_{j}+U^{T}\right)+Y_{j}+Y_{j}^{T}+Q \\
\Psi_{i j}^{(12)}=\sum_{k=1}^{r-1} \beta_{k}\left(W_{k}-W_{r}\right)+\left(P_{j}+U\right) A_{\tau_{i}}+A_{i}^{T} W_{j}-Y_{j}+Y_{j}^{T} \\
\Psi_{i j}^{(22)}=\sum_{k=1}^{r-1} \beta_{k}\left(S_{k}-S_{r}\right)-T_{j}-T_{j}^{T}+W_{j}^{T} A_{\tau_{i}}+A_{\tau_{i}}^{T} W_{j}-Q .
\end{gathered}
$$

\section{Main Results}

Consider the open-loop system (2). The aim of this section is to find conditions for the stability of the unforced time-delay T-S fuzzy system by using the LyapunovKrasovskii theory.

\section{Theorem 1}

Under assumption 1, the time-delay Takagi-Sugeno fuzzy system is stable if there exist positive definite symmetric matrices $P_{j} \succ 0, Q \succ 0, Z \succ 0, \quad X=X^{T}$, $Y_{j}$ and $T_{j} j=1,2, \cdots, r$, such that the following LMIs hold.

$$
\begin{gathered}
P_{k}+X \succ 0, \quad k \in\{1, \cdots, r\} \\
\Omega_{i j}+\Omega_{j i} \prec 0, \quad i \leq j,
\end{gathered}
$$

where

$\Omega_{i j}=$

$$
\left[\begin{array}{cccc}
\sum_{k=1}^{r} \beta_{k}\left(P_{k}+X\right) & & & \\
+P_{j} A_{i}+A_{i}^{T} P_{j} & P_{j} A_{\tau i}-Y_{j}+T_{j}^{T} & \tau_{0} A_{i}^{T} Z & -\tau_{0} Y_{j} \\
+Y_{j}+Y_{j}^{T}+Q & & & \\
* & -T_{j}-T_{j}^{T}-(1-d) Q & \tau_{0} A_{\tau i}^{T} Z & -\tau_{0} T_{j} \\
* & * & -\tau_{0} Z & 0 \\
* & * & * & -\tau_{0} Z
\end{array}\right]
$$

Proof

Let consider the fuzzy weighting-dependent LyapunovKrasovskii functional as

$$
V\left(x_{t}\right)=V_{1}+V_{2}+V_{3}
$$

where $x_{t}=x(t+\alpha), \alpha \in[-\tau, 0]$, and

$$
\begin{gathered}
V_{1}=x^{T}(t)\left(\sum_{j=1}^{r} h_{j}(t) P_{j}\right) x(t) \\
V_{2}=\int_{t-\tau(t)}^{t} x^{T}(s) Q x(s) \mathrm{d} s \\
V_{3}=\int_{-\tau_{0}}^{0} \int_{t+\theta}^{t} \dot{x}(s)^{T} Z \dot{x}(s) \mathrm{d} s \mathrm{~d} \theta .
\end{gathered}
$$

The Newton-Leibniz formula gives

$$
x(t-\mathrm{d}(t))=x(t)-\int_{t-\mathrm{d}(t)}^{t} \dot{x}(s) \mathrm{d} s
$$

which yields

$$
\begin{aligned}
& 2\left[x(t)^{T} Y(t)+x(t-\tau(t))^{T} T(t)\right] \\
& \times\left[x(t)-x(t-\tau(t))-\int_{t-\tau(t)}^{t} \dot{x}(s) \mathrm{d} s\right] \equiv 0
\end{aligned}
$$

where $Y(t)=\sum_{j=1}^{r} h_{j}(t) Y_{j}$ and $T(t)=\sum_{j=1}^{r} h_{j}(t) T_{j}$ and where $Y_{j}$ and $T_{j}$ are arbitrary matrices with appropriate dimensions, $j=1,2, \cdots, r$. With (14), the time derivative of $V\left(x_{t}\right)$ with respect to $t$ along solutions to (2) is given by:

$$
\begin{aligned}
& \dot{V}\left(x_{t}\right) \\
& =x(t)^{T}\left(\sum_{j=1}^{r} \dot{h}_{j}(t) P_{j}\right) x(t)+2 x(t)^{T}\left(\sum_{j=1}^{r} h_{j}(t) P_{j}\right) \dot{x}(t) \\
& +x(t)^{T} Q x(t)-(1-\dot{\tau}(t)) x(t-\tau(t))^{T} Q x(t-\tau(t)) \\
& +\tau_{0} \dot{x}(t)^{T} Z \dot{x}(t)-\int_{t-\tau_{0}}^{t} \dot{x}(s)^{T} Z \dot{x}(s) \mathrm{d} s \\
& \leq \sum_{k=1}^{r} \dot{h}_{k}(t) x(t)^{T} P_{k} x(t)+2 x(t)^{T}\left(\sum_{j=1}^{r} h_{j}(t) P_{j}\right) \dot{x}(t) \\
& +x(t)^{T} Q(t) x(t)-(1-d) x(t-\tau(t))^{T} Q x(t-\tau(t)) \\
& +\tau_{0} \dot{x}(t)^{T} Z \dot{x}(t)-\int_{t-\tau_{0}}^{t} \dot{x}(s)^{T} Z \dot{x}(s) \mathrm{d} s \\
& +2\left[x(t)^{T} Y(t)+x(t-\tau(t))^{T} T(t)\right] \\
& \quad \times\left[x(t)-x(t-\tau(t))-\int_{t-\tau(t)}^{t} \dot{x}(s) \mathrm{d} s\right]
\end{aligned}
$$

where the inequality is caused only by $\dot{\tau}(t) \leq d$ and $\tau(t) \leq \tau_{0}$.

Based on (5), it follows that $\sum_{k=1}^{r} \dot{h}_{k} X=\bar{X}=0$, where $X$ symmetric matrix of proper dimension. Adding $\bar{X}$ to (15), and by substitution of $\dot{x}(t)$ with state Equation (2), we have

$$
\begin{aligned}
\dot{V}\left(x_{t}\right) & \leq \sum_{k=1}^{r} \dot{h}_{k}(t) x(t)^{T}\left(P_{k}+X\right) x(t) \\
+ & x(t)^{T} P(t)\left[A(t) x(t)+A_{\tau}(t) x(t-\tau(t))\right] \\
+ & {\left[x(t)^{T} A^{T}(t)+x^{T}(t-\tau(t)) A_{\tau}^{T}(t)\right] P(t) x(t) } \\
+ & x(t)^{T} Q(t) x(t)-(1-d) x(t-\tau(t))^{T} Q x(t-\tau(t)) \\
+ & \tau_{0} \dot{x}(t)^{T} Z \dot{x}(t)-\int_{t-\tau_{0}}^{t} \dot{x}(s)^{T} Z \dot{x}(s) \mathrm{d} s \\
+ & 2\left[x(t)^{T} Y(t)+x(t-\tau(t))^{T} T(t)\right] \\
& \times\left[x(t)-x(t-\tau(t))-\int_{t-\tau(t)}^{t} \dot{x}(s) \mathrm{d} s\right]
\end{aligned}
$$

Then, based on assumption 1 and if (8) holds, (15) yields to 


$$
\begin{aligned}
& \dot{V}\left(x_{t}\right) \leq \eta(t)^{T}\left[\tilde{\Omega}(t)+\tau_{0} M(t) Z^{-1} M(t)^{T}\right] \eta(t) \\
&-\int_{t-\tau_{0}}^{t}\left[\eta(t)^{T} M(t)+\dot{x}(s)^{T} Z\right] \\
& \cdot Z^{-1}\left[M(t)^{T} \eta(t)+Z \dot{x}(s)\right] \mathrm{d} s
\end{aligned}
$$

$$
\begin{aligned}
& \text { where } \eta(t)^{T}=\left[x(t)^{T}, x(t-\tau(t))^{T}\right], \\
& M(t)^{T}=\left[Y(t)^{T}, T(t)^{T}\right] \text { and }
\end{aligned}
$$

$$
\tilde{\Omega}(t)=\left[\begin{array}{cc}
P_{\beta}+P(t) A(t)+A(t)^{T} P(t) & \\
+Y(t)+Y(t)^{T}+Q & +\tau_{0} A(t)^{T} Z A_{\tau}(t)-Y(t)+T(t)^{T} \\
+\tau_{0} A(t)^{T} Z A(t) & -T(t)-T(t)^{T}-(1-d) Q \\
* & +\tau_{0} A_{\tau}(t)^{T} Z A_{\tau}(t)
\end{array}\right]
$$

where $P_{\beta}=\sum_{k=1}^{r} \beta_{k}\left(P_{k}+X\right), \quad P(t)=\sum_{j=1}^{r} h_{j}(z(t)) P_{j}, \quad A(t)=\sum_{i=1}^{r} h_{i}(z(t)) A_{i}$ and $A_{\tau}(t)=\sum_{j=1}^{r} h_{j}(z(t)) A_{\tau j}$ if (9) holds, that implies

$$
\begin{gathered}
\Omega(t)=\left[\begin{array}{cccc}
\sum_{k=1}^{r} \beta_{k}\left(P_{k}+X\right) & & & \\
+\sum_{i, j=1}^{r} h_{j}(t) h_{i}(t) P_{j} A_{i} & P(t) A_{\tau}(t) & \tau_{0} A(t)^{T} Z & -\tau_{0} Y(t) \\
+Y(t)+Y^{T}(t)+Q & -Y(t)-T^{T}(t) & & \\
+\tau_{0} A(t)^{T} Z A(t) & & & \\
* & -T(t)-T(t)^{T} & \tau_{0} A_{\tau}(t)^{T} Z & -\tau_{0} T(t) \\
* & -(1-d) Q & -\tau_{0} Z & 0 \\
* & * & * & -\tau_{0} Z
\end{array}\right] \\
=\sum_{j=1}^{r} \sum_{i=1}^{r} h_{j}(t) h_{i}(t) \Omega_{i j}=\frac{1}{2} \sum_{j=1}^{r} \sum_{i=1}^{r} h_{j}(t) h_{i}(t)\left(\Omega_{i j}+\Omega_{j i}\right) \prec 0, \\
\end{gathered}
$$

which is equivalent to $\tilde{\Omega}(t)+\tau_{0} M(t) Z^{-1} M(t)^{T} \prec 0$ by Schur complement. Therefore, from (16) we have $\dot{V}\left(x_{t}\right) \prec 0$ and (2) is stable.

\section{Further Fuzzy Weighting-Dependent Lyapunov-Krasovskii Functional Methods}

In this section, we give a less conservative fuzzy weighting-dependent Lyapunov-Krasovskii functional. The main development is stated as follows.

\section{Theorem 2}

Under assumption 1, and for $0 \leq \varepsilon \leq 1$ the time-delay Takagi-Sugeno fuzzy system is stable if there exist positive definite symmetric matrices $P_{j} \succ 0, Q \succ 0, Z \succ 0$, $X_{1}=X_{1}^{T}, \quad X_{2}=X_{2}{ }^{T}, \quad Y_{j}$ and $T_{j}, \quad j=1,2, \cdots, r$, such that the following LMIs hold.

$$
\begin{gathered}
P_{k}+X_{1}+\varepsilon X_{2} \succ 0, \quad k \in\{1, \cdots, r\} \\
P_{j}+\varepsilon X_{1} \succ 0, \quad j=1,2, \cdots, r \\
\Omega_{i j}+\Omega_{j i} \prec 0, \quad i \leq j,
\end{gathered}
$$

where

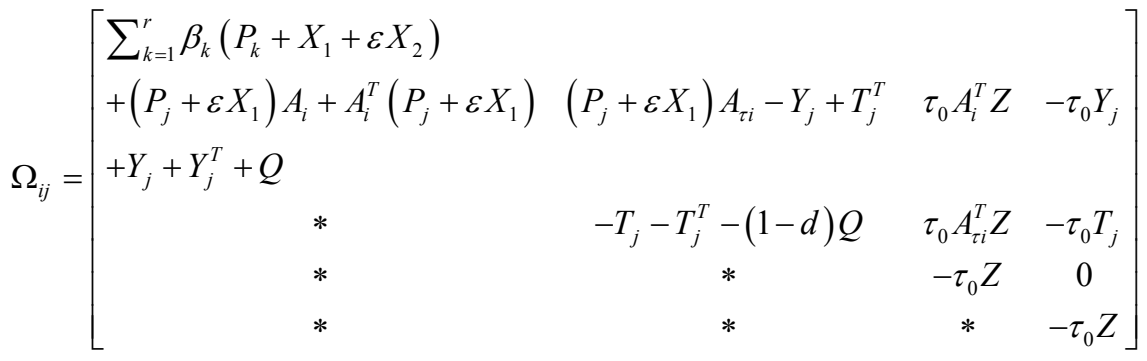




\section{Proof}

Let consider the fuzzy weighting-dependent LyapunovKrasovskii functional as

$$
V\left(x_{t}\right)=V_{1}+V_{2}+V_{3}
$$

where $x_{t}=x(t+\alpha), \alpha \in[-\tau, 0]$, and

$$
\begin{gathered}
V_{1}=x^{T}(t)\left(\sum_{j=1}^{r} h_{j}(t)\left(P_{j}+\varepsilon X_{1}\right)\right) x(t) \\
V_{2}=\int_{t-\tau(t)}^{t} x^{T}(s) Q x(s) \mathrm{d} s \\
V_{3}=\int_{-\tau_{0}}^{0} \int_{t+\theta}^{t} \dot{x}(s)^{T} Z \dot{x}(s) \mathrm{d} s \mathrm{~d} \theta .
\end{gathered}
$$

The Newton-Leibniz formula gives

$$
x(t-d(t))=x(t)-\int_{t-d(t)}^{t} \dot{x}(s) \mathrm{d} s,
$$

which yields

$$
\begin{aligned}
& 2\left[x(t)^{T} Y(t)+x(t-\tau(t))^{T} T(t)\right] \\
& \times\left[x(t)-x(t-\tau(t))-\int_{t-\tau(t)}^{t} \dot{x}(s) \mathrm{d} s\right] \equiv 0
\end{aligned}
$$

where $Y(t)=\sum_{j=1}^{r} h_{j}(t) Y_{j} \quad$ and $\quad T(t)=\sum_{j=1}^{r} h_{j}(t) T_{j}$ and where $Y_{j}$ and $T_{j}$ are arbitrary matrices with appropriate dimensions, $j=1,2, \cdots, r$. With (14), the time derivative of $V\left(x_{t}\right)$ with respect to $t$ along solutions to (2) is given by:

$$
\begin{aligned}
\dot{V}\left(x_{t}\right) & =x(t)^{T}\left(\sum_{j=1}^{r} \dot{h}_{j}(t)\left(P_{j}+\varepsilon X_{1}\right)\right) x(t) \\
& +2 x(t)^{T}\left(\sum_{j=1}^{r} h_{j}(t)\left(P_{j}+\varepsilon X_{1}\right)\right) \dot{x}(t) \\
& +x(t)^{T} Q x(t)-(1-\dot{\tau}(t)) x(t-\tau(t))^{T} Q x(t-\tau(t)) \\
& +\tau_{0} \dot{x}(t)^{T} Z \dot{x}(t)-\int_{t-\tau_{0}}^{t} \dot{x}(s)^{T} Z \dot{x}(s) \mathrm{d} s \\
\leq & \sum_{k=1}^{r} \dot{h}_{k}(t) x(t)^{T}\left(P_{k}+\varepsilon X_{1}\right) x(t) \\
+ & 2 x(t)^{T}\left(\sum_{j=1}^{r} h_{j}(t)\left(P_{j}+\varepsilon X_{1}\right)\right) \dot{x}(t) \\
+ & x(t)^{T} Q(t) x(t)-(1-d) x(t-\tau(t))^{T} Q x(t-\tau(t)) \\
+ & \tau_{0} \dot{x}(t)^{T} Z \dot{x}(t)-\int_{t-\tau_{0}}^{t} \dot{x}(s)^{T} Z \dot{x}(s) \mathrm{d} s \\
+ & 2\left[x(t)^{T} Y(t)+x(t-\tau(t))^{T} T(t)\right] \\
& \times\left[x(t)-x(t-\tau(t))-\int_{t-\tau(t)}^{t} \dot{x}(s) \mathrm{d} s\right]
\end{aligned}
$$

where the inequality is caused only by $\dot{\tau}(t) \leq d$ and $\tau(t) \leq \tau_{0}$.

Based on (5), it follows that

$\sum_{k=1}^{r} \dot{h}_{k}(1-\varepsilon) X_{1}=\bar{X}_{1}=0$, and $\sum_{k=1}^{r} \dot{h}_{k} \varepsilon X_{2}=\bar{X}_{2}=0$, where $\bar{X}_{1}$ and $\bar{X}_{2}$ symmetric matrices of proper dimension. Adding $\bar{X}_{1}$ and $\bar{X}_{2}$ to (15), and by substitution of $\dot{x}(t)$ with state Equation (2), we have

$$
\begin{aligned}
& \dot{V}\left(x_{t}\right) \leq \sum_{k=1}^{r} \dot{h}_{k}(t) x(t)^{T}\left(P_{k}+X_{1}+\varepsilon X_{2}\right) \\
& +x(t)^{T} \tilde{P}(t)\left[A(t) x(t)+A_{\tau}(t) x(t-\tau(t))\right] \\
& +\left[x(t)^{T} A^{T}(t)+x^{T}(t-\tau(t)) A_{\tau}^{T}(t)\right] \tilde{P}(t) x(t) \\
& +x(t)^{T} Q(t) x(t)-(1-d) x(t-\tau(t))^{T} Q x(t-\tau(t)) \\
& +\tau_{0} \dot{x}(t)^{T} Z \dot{x}(t)-\int_{t-\tau_{0}}^{t} \dot{x}(s)^{T} Z \dot{x}(s) \mathrm{d} s \\
& +2\left[x(t)^{T} Y(t)+x(t-\tau(t))^{T} T(t)\right] \\
& \times\left[x(t)-x(t-\tau(t))-\int_{t-\tau(t)}^{t} \dot{x}(s) \mathrm{d} s\right]
\end{aligned}
$$

where

$$
\begin{gathered}
\tilde{P}(t)=\sum_{k=1}^{r} h_{k}(z(t))\left(P_{k}+\varepsilon X_{1}\right), \\
A(t)=\sum_{i=1}^{r} h_{i}(z(t)) A_{i}, \quad \text { and } \\
A_{\tau}(t)=\sum_{j=1}^{r} h_{j}(z(t)) A_{\tau j} .
\end{gathered}
$$

Then, based on assumption 1 and if (8) holds, (15) yields to

$$
\begin{aligned}
& \dot{V}\left(x_{t}\right) \leq \eta(t)^{T}\left[\tilde{\Omega}(t)+\tau_{0} M(t) Z^{-1} M(t)^{T}\right] \eta(t) \\
&-\int_{t-\tau_{0}}^{t}\left[\eta(t)^{T} M(t)+\dot{x}(s)^{T} Z\right] \\
& \cdot Z^{-1}\left[M(t)^{T} \eta(t)+Z \dot{x}(s)\right] \mathrm{d} s
\end{aligned}
$$

where $\eta(t)^{T}=\left[x(t)^{T}, x(t-\tau(t))^{T}\right]$,

$M(t)^{T}=\left[Y(t)^{T}, T(t)^{T}\right]$ and

$\tilde{\Omega}(t)=$

$$
\left[\begin{array}{cl}
\tilde{P}_{\beta}+\tilde{P}(t) A(t)+A(t)^{T} \tilde{P}(t) & \\
+Y(t)+Y(t)^{T}+Q & \tilde{P}(t) A_{\tau}(t)-Y(t)+T(t)^{T} \\
+\tau_{0} A(t)^{T} Z A(t) & +\tau_{0} A(t)^{T} Z A_{\tau}(t) \\
& \\
& -T(t)-T(t)^{T}-(1-d) Q \\
& +\tau_{0} A_{\tau}(t)^{T} Z A_{\tau}(t)
\end{array}\right]
$$

where $\tilde{P}_{\beta}=\sum_{k=1}^{r} \beta_{k}\left(P_{k}+X_{1}+\varepsilon X_{2}\right)$

if (9) holds, that implies 


$$
\begin{aligned}
\Omega(t) & =\left[\begin{array}{cccc}
\tilde{P}_{\beta}+\sum_{i, j=1}^{r} h_{j}(t) h_{i}(t)\left(P_{j}+\varepsilon X_{1}\right) A_{i} & \tilde{P}(t) A_{\tau}(t) & \tau_{0} A(t)^{T} Z & -\tau_{0} Y(t) \\
+Y(t)+Y^{T}(t)+Q+\tau_{0} A(t)^{T} Z A(t) & -Y(t)-T^{T}(t) & & \\
* & -T(t)-T(t)^{T} & \tau_{0} A_{\tau}(t)^{T} Z & -\tau_{0} T(t) \\
* & -(1-d) Q & -\tau_{0} Z & 0 \\
* & * & * & -\tau_{0} Z
\end{array}\right] \\
& =\sum_{j=1}^{r} \sum_{i=1}^{r} h_{j}(t) h_{i}(t) \Omega_{i j} \\
& =\frac{1}{2} \sum_{j=1}^{r} \sum_{i=1}^{r} h_{j}(t) h_{i}(t)\left(\Omega_{i j}+\Omega_{j i}\right) \\
& \prec 0,
\end{aligned}
$$

which is equivalent to $\tilde{\Omega}(t)+\tau_{0} M(t) Z^{-1} M(t)^{T} \prec 0$ by Schur complement. Therefore, from (16) we have $\dot{V}\left(x_{t}\right) \prec 0$ and (2) is stable.

\section{Numerical Examples}

In order to show the improvements of proposed approaches over some existing results, in this section, we present two numerical examples, which concern the feasibility of a time delay T-S fuzzy system. Indeed, we compare our fuzzy weighting-dependent Lyapunov-Krasovskii approach (Theorem 1 and Theorem 2) with the Lemma 1 in [2].

Example 1. Consider the following T-S fuzzy system with time-delay:

$$
\begin{aligned}
& \dot{x}(t)=\sum_{i=1}^{r} h_{i}(z(t))\left\{A_{i} x(t)+A_{\tau i} x(t-\tau(t))\right\} \\
& x(t)=\phi(t), \quad t \in\left[-\tau_{0}, 0\right],
\end{aligned}
$$

with: $r=2$

$$
\begin{aligned}
& A_{1}=\left[\begin{array}{cc}
-3.2 & 0.6 \\
0 & -2.1
\end{array}\right], A_{2}=\left[\begin{array}{cc}
-1 & 0 \\
1 & -3
\end{array}\right], A_{\tau 1}=\left[\begin{array}{cc}
1 & 0.9 \\
0 & 2
\end{array}\right], \\
& A_{\tau 2}=\left[\begin{array}{cc}
0.9 & 0 \\
1 & 1.6
\end{array}\right] .
\end{aligned}
$$

The purpose is to compute the maximum value of $\tau_{0}$ such that the fuzzy system is stable. Table 1 lists the computation results using delay-dependent conditions in Theorem1, and in Theorem $2(\varepsilon=0.1)$ compared with the existing delay-dependent method in Lemma1 in [2], for different values of $d$. It is seen from Table 1 that the largest values of $\tau_{0}$ are obtain by using our methods. (29).

Example 2. Consider the time delay T-S fuzzy system with: $r=2$

$$
\begin{aligned}
& A_{1}=\left[\begin{array}{ll}
-5 & -4 \\
-1 & -2
\end{array}\right], A_{2}=\left[\begin{array}{ll}
-2 & -4 \\
20 & -2
\end{array}\right], \quad A_{\tau 1}=\left[\begin{array}{cc}
0.5 & 0.6 \\
1 & 0.4
\end{array}\right], \\
& A_{\tau 2}=\left[\begin{array}{cc}
0.3 & -0.4 \\
0.5 & 0
\end{array}\right] .
\end{aligned}
$$

This example shows that the stability cannot be tested by quadratic methods while can be verified by our fuzzy weighting-dependent methods in Theorems.

The purpose is to compute the maximum value of $\tau_{0}$ such that the fuzzy system is asymptotically stable.

Table 2 lists the computation results for different values of $d$ under the same upper bound $\beta_{i} \leq \beta=0.8$. It reveals from Table 2 that Theorem 1 and Theorem 2 with $\varepsilon=0.1$ produces better results than Lemma 1 and Lemma 2.

\section{Conclusions}

This paper provided new conditions for Delay-dependent

Table 1. Comparison results of maximum $\tau_{0}$ for Example 1.

\begin{tabular}{cccccc}
\hline \multirow{4}{*}{$\beta=1$} & Methods & $\boldsymbol{d = 0}$ & $\boldsymbol{d}=\mathbf{0 . 0 2}$ & $\boldsymbol{d}=\mathbf{0 . 1}$ & $\boldsymbol{d}=\mathbf{0 . 9}$ \\
\hline \multirow{6}{*}{$\beta=0.5$} & Lemma 1 & 0.6185 & 0.5618 & 0.4809 & 0.4513 \\
& Theorem 1 & 0.6185 & 0.5618 & 0.4810 & 0.4530 \\
& Theorem 2 & $+\infty$ & $+\infty$ & 0.5500 & 0.4689 \\
& Lemma 1 & 0.6248 & 0.5630 & 0.4814 & 0.4537 \\
& Theorem 1 & 0.6248 & 0.5630 & 0.4817 & 0.4562 \\
& Theorem 2 & $+\infty$ & $+\infty$ & 0.5500 & 0.4695 \\
\hline
\end{tabular}

Table 2. Comparison results of maximum $\tau_{0}$ for Example 2.

\begin{tabular}{ccccc}
\hline & Methods & $\boldsymbol{d}=\mathbf{0}$ & $\boldsymbol{d}=\mathbf{0 . 5}$ & $\boldsymbol{d}=\mathbf{0 . 9}$ \\
\hline \multirow{4}{*}{$\beta=0.8$} & Lemma 1 & 0.3883 & 0.3225 & 0.2518 \\
& Lemma 2 & $+\infty$ & 0.5221 & 0.2844 \\
& Theorem 1 & $+\infty$ & 0.6933 & 0.3058 \\
& Theorem 2 & $+\infty$ & $+\infty$ & 0.8133 \\
\hline
\end{tabular}


stability problems of time-delay Takagi-Sugeno fuzzy systems in terms of a combination of the LMI approach and the use of fuzzy weighting-dependent LyapunovKrasovskii functionals.

The stability conditions proposed in this note are less conservative than some of those in the literature, which has been illustrated via examples.

\section{References}

[1] Y. Y. Cao and P. M. Frank, "Stability Analysis and Synthesis of Nonlinear Time-Delay Systems via TakagiSugeno Fuzzy Models," Fuzzy Sets and Systems, Vol. 124 No. 2, 2001, pp. 213-229. doi:10.1016/S0165-0114(00)00120-2

[2] C. Lin, Q. G. Wang and T. H. Lee, "Delay-Dependent LMI Conditions for Stability and Stabilization of T-S Fuzzy Systems with Bounded Time-Delay," Fuzzy Sets and Systems, Vol. 157 No. 9, 2006, pp. 1229-1247. doi:10.1016/j.fss.2005.10.001

[3] C. Lin, Q. G. Wang and T. H. Lee, "Fuzzy WeightingDependent Approach to $H_{\infty}$ Filter Design for Time-Delay Fuzzy Systems," IEEE Transactions on Signal Processing, Vol. 55 No. 6, 2007, pp. 2746-2751. doi:10.1109/TSP.2007.893761

[4] C. Lin, Q. G. Wang and T. H. Lee, "LMI Approach to Analysis and Control of Takagi-Sugeno Fuzzy Systems with Time Delay,” Springer-Verlag, Berlin, 2007.

[5] Y. Y. Cao and P. M. Frank. "Analysis and Synthesis of Nonlinear Time-Delay Systems via Fuzzy Control Approach," IEEE Transactions on Fuzzy Systems, Vol. 8, No. 2, 2000, pp. 200-211. doi:10.1109/91.842153

[6] K. Tanaka, T. Hori, and H. O. Wang, "A multiple Lyapunov Function Approach to Stabilization of Fuzzy Control Systems," IEEE Transactions on Fuzzy Systems, Vol. 11 No. 4, 2003, pp. 582-589. doi:10.1109/TFUZZ.2003.814861

[7] K. Tanaka and H. O. Wang, "Fuzzy Control Systems Design and Analysis: A Linear Matrix Inequality Approach," John Wiley and Sons, Hoboken, 2001.

[8] L. A. Mozelli, R. M. Palhares, F. O. Souza and E. M.
Mendes, "Reducing Conservativeness in Recent Stability Conditions of TS Fuzzy Systems," Automatica, Vol. 45, No. 6, 2009, pp. 1580-1583. doi:10.1016/j.automatica.2009.02.023

[9] T. Takagi and M. Sugeno, "Fuzzy Identification of Systems and Its Application to Modeling and Control," IEEE Transactions on System, Man and Cybernetics, Vol. 15 No. 1, 1985, pp. 116-132.

[10] L. K. Wong, F. H. F. Leung and P. K. S. Tam, "Stability Design of TS Model Based Fuzzy Systems," Proceedings of the 6th IEEE International Conference on Fuzzy Systems, Barcelona, 1-5 July 1997, pp. 83-86. doi:10.1109/FUZZY.1997.616349

[11] C. H. Fang, Y. S. Liu, S. W. Kau, L. Hong and C. H. Lee, "A New LMI-Based Approach to Relaxed Quadratic Stabilization of T-S Fuzzy Control Systems," IEEE Transactions on Fuzzy Systems, Vol. 14, No. 3, 2006, pp. 386397.

[12] H. O. Wang, K. Tanaka and M. F. Griffin, “An Approach to Fuzzy Control of Nonlinear Systems: Stability and Design Issues," IEEE Transactions on Fuzzy Systems, Vol. 4, No. 1, 1996, pp. 14-23. doi:10.1109/91.481841

[13] K. Tanaka, T. Hori and H. O. Wang, "A Fuzzy Lyapunov Approach to Fuzzy Control System Design," Proceedings of the 2001 American Control Conference, Arlington, 25-27 June 2001, pp. 4790-4795.

[14] M. A. L. Thathachar and P. Viswanah, "On the Stability of Fuzzy Systems," IEEE Transactions on Fuzzy Systems, Vol. 5, No. 1, 1997, pp. 145-151. doi:10.1109/91.554461

[15] C. W. Chen, "Stability Conditions of Fuzzy Systems and Its Application to Structural and Mechanical Systems," Advances in Engineering Software, Vol. 37, No. 9, 2006, pp. 624-629. doi:10.1016/j.advengsoft.2005.12.002

[16] S. Boyd, L. Ghaoui, E. Feron and V. Balakrishnan, "Linear Matrix Inequalities in Systems and Control Theory," SIAM, Philadelphia, 1994. doi:10.1137/1.9781611970777

[17] Y. Manai and M. Benrejeb, "Stability for Continuous Takagi-Sugeno Fuzzy System Based on Fuzzy Lyapunov Function," 2011 International Conference on Communications, Computing and Control Applications, Hammamet, 3-5 March 2011. doi:10.1109/CCCA.2011.6031521 